seroconversion, a key stage to $\mathrm{T} 1 \mathrm{D}$, the conventional analysis found some microbiota with changed abundance after the occurrence of seroconversion (figure 1a). Meanwhile, our analysis further show that a lot of microbiota actually have great abundance variance change before seroconversion (figure $1 \mathrm{~b}$ ), which can even be efficient features to classify the healthy and seroconversion individuals with about $80 \%$ accuracy (figure 1c). More importantly, this microbiota with critical abundance variance as key species is also associated with T1D clinical antibody or even some new (figure 1d).

Conclusions Dissimilar to common biomarkers like a clinical antibody, the individual specific signatures, e.g. variance of gut microbiota abundance, would be an alternative approach for personalised pre-disease or early-disease diagnosis.

\section{IDDF2018-ABS-0161 GUT MICROBIOME ACROSS STAGES OF HBV INFECTION}

Zhangran Chen*, Yurou Xie, Chuanxing Xiao, Xiang Zhang, Jianlin Ren. Institute for Microbial Ecology, Medical College of Xiamen University, China

\subsection{6/gutjnl-2018-IDDFabstracts. 18}

Background Increasing evidence suggests that the gut microbiota has evolved as a new important player in the pathogenesis of hepatitis B virus-induced chronic liver disease, including chronic hepatitis, cirrhosis. However, the composition and structure alteration of the gut microbiota associated with the stage and progression of $\mathrm{HBV}$ infection remains unknown. Hence, the aim of the study is to elucidate the microbial influence which contributes to the microbial shift of $\mathrm{HBV}$ patients in different stages.

Methods All subjects enrolled had clinic test performed in the Zhongshan Hospital, Xiamen University. A total of 62 subjects were finally selected for inclusion in this study, including 7 healthy individuals, $13 \mathrm{HBeAg}$ negative chronic $\mathrm{HBV}$ infection patients (HNCHI), $6 \mathrm{HBeAg}$ negative chronic hepatitis B patients (HNCHB), $5 \mathrm{HBeAg}$ positive chronic hepatitis $\mathrm{B}$ patients (HPCHB) and 31 decompensated cirrhosis after hepatitis $\mathrm{B}$ patients (DCAHB) matched in age, gender and body mass index (BMI). Stools from all subjects were collected to extract DNA, then for $16 \mathrm{~S}$ rDNA high-throughput sequencing and further compared by the bacterial diversity, community and structure, differential taxa analysis.

Results DCAHB patients showed decreased gut bacterial diversity and more unique OTU numbers compared to the other four groups, suggesting that DCAHB is distinctive, which could further be confirmed by the PCA analysis. In phyla level, the relative abundance of Fusobacteria and Proteobacteria increased while Firmicutes content decreased from the health, HNCHI, HNCHB, HPCHB to DCAHB group. In genus level, the high level of Streptococcus, Veillonella, Enterobacteriaceae, Lactobacillus and Haemophilus, while decreased Clostridiaceae, Phascolarctobacterium, Coprococcus and Ruminococcaceae in DCAHB conform the disease progression state. In addition, LEfSe analysis reveals that Bacteroides fragilis, Bacteroides eggerthii, Veillonella dispar, Streptococcus anginosus, Corprococcus eutactus and Akkermansia muciniphila could serve as target microorganism for the further investigation.

Conclusions These compositional and structural changes in the gut microbiota in varied stage HBV patients suggest the potential contributions of gut microbiota to the progression of $\mathrm{HBV}$ infection and thus provide new insight into gut microbiota-targeted interventions to improve the prognosis of this disease.

\section{IDDF2018-ABS-0162 ALTERED GUT MICROBIOTA IN EXPERIMENTAL MOUSE MODELS OF NON- ALCOHOLIC FATTY LIVER DISEASE}

Bangzhou Zhang*, Lina Fan, Chuanxing Xiao, Jianlin Ren. Department of Gastroenterology, Zhongshan Hospital Affiliated to Xiamen University, China

\subsection{6/gutjnl-2018-IDDFabstracts. 19}

Background Non-alcoholic fatty liver disease (NAFLD) has become the most common liver disease worldwide and is thought to be strongly associated with gut microbiota. Several diet models were therefore built in mice to try to clarify the molecular mechanisms. However, how and to what extent these diet models alter the composition of the gut microbiota have not yet been clearly elucidated.

Methods In this study, we developed three mouse models of NAFLD using methionine-choline-deficient (MCD) diet, highfat (HF) diet, and choline-deficient- high-fat (CD-HF) diet, evaluated the severity of steatohepatitis and sequenced the faecal bacteria by targeting $16 \mathrm{~S}$ V4-V6 regions on Illumina MiSeq using PE 300 reagents.

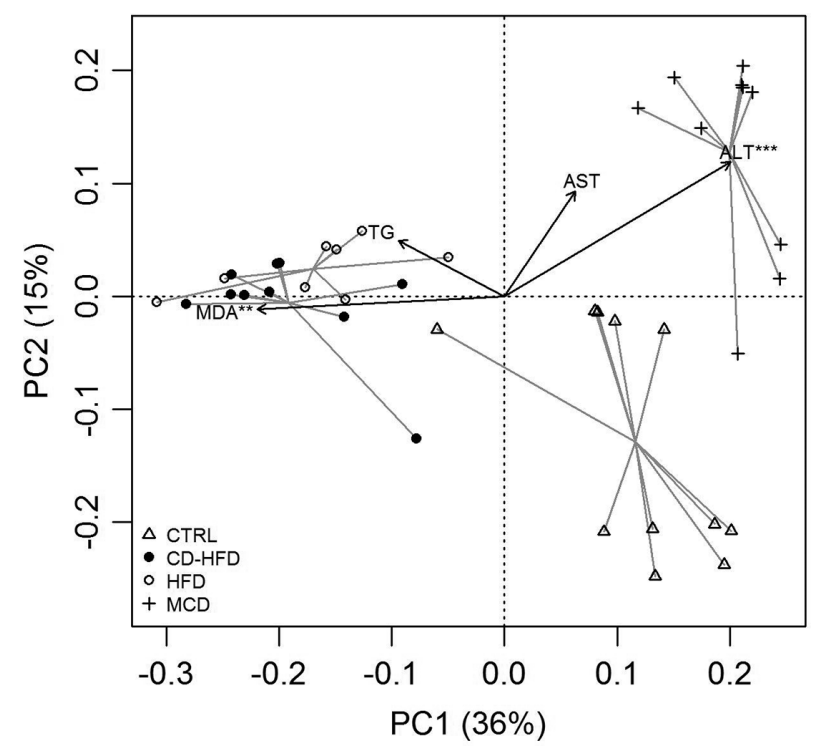

Abstract IDDF2018-ABS-0162 Figure 1 PCA ordination of the gut microbiota communities, fitted with serum and liver tissue biochemistry assays $\left({ }^{* *} \mathrm{P}<0.001,{ }^{* *} \mathrm{P}<0.01\right)$

Results Histological scores showed that MCD induced the severest steatohepatitis, followed by $\mathrm{HF}$ and CD-HF diets. Based on operational taxonomic units (OTUs) at cutoff of 97\% similarity, there were significant (PERMANOVA, $\mathrm{p}=0.001$ ) differences in overall gut bacterial communities among MCD, HF, CD-HFD, and the Control, forming three major clusters in PCA ordination with HF and CD-HF groups more similar (figure 1). Furthermore, $\alpha$-diversity of $\mathrm{HF}$ and CD-HF groups, including observed OTU numbers, Shannon index, and Pielou evenness were significantly (ANOVA, $\mathrm{p}<0.05)$ higher than the Control and MCD group. Overall, 
mouse gut bacteria were mainly composed of Fimicutes (Ruminococcaceae, Lachnospiraceae, Clostridiaceae) and Bacteroidetes (S24-7, Porphyromonadaceae). More specifically, Ruminococcus was significantly reduced in the three NAFLD models than the Control and was identified as the biomarker of NAFLD in LEfSe analysis. More biomarkers at genus level (Lachnospira, S24-7, etc.) were identified in pairwise comparison of one mice model with the Control.

Conclusions In summary, the composition of gut microbiota varied remarkably between mice administrated different experimental diets to induce non-alcoholic fatty liver disease.

\section{IDDF2018-ABS-0167 THE TCF3/MIR-145-5P/LINC00113 POSITIVE FEEDBACK LOOP PROMOTES COLORECTAL CANCER PROGRESSION BY SILENCING STAT1}

Changsheng Yan*. Zhongshan Hospital Xiamen University, China

\subsection{6/gutjnl-2018-IDDFabstracts.20}

Background To identify the role of LINC00113 in colorectal cancer.

Methods Jaspar and Cor $\mathrm{R}$ package was used to predict the potential translational factor of LINC00113. CHIP and dual luciferase report assay were enrolled to confirm the binding sites between LINC00113 and translational factor. Besides, dual luciferase report assay was used to confirm the binding site of LINC00113, miRNA and TCF3. Flow cytometry was used to detect the cell cycle and apoptosis. RIP assay was used to confirm the binding of LINC00113 and DNMT1.

Results Using the Gene expression omibus (GEO) data set and bioinformatics analyses, we identified LINC00113 as an independent risk prognostic factor in colorectal cancer. To identify the mechanism how LINC00113 in colorectal cancer, we firstly to seek the translational factor which could up-regulate the expression of LINC00113. We found that the expression level of TCF3 was strongly positively correlated with LINC0113. Besides, knockdown and overexpress of TCF3 could coordinately lead to the alteration of LINC00113. What's more, CHIP and luciferase assay confirm that TCF3 binds to the promoter sequence of LINC00113 and thus promoting the expression of LINC00113. Then we found that LINC00113 might be a negative regulator of miR-145-5 $\mathrm{p}$ by loss- and gain-of-function assay. Besides, we found that miR$149-5 \mathrm{p}$ had the potential ability binding to the 3'-UTR of TCF3, thus leading to the degradation of TCF3. However, as the increased level of LINC00113, the expression level of miR-145-5 p was lowly expressed in colorectal cancer, which leads to the expression level of TCF3 significantly up-regulated in colorectal cancer. At last, the high level of TCF3 in turn promotes the expression of LINC00113. Moreover, we also found that LINC00113 could bind to DNMT1 to promote the methylation of promoter sequence of STAT1 and leading to the down-regulation of STAT1, which act as a tumour suppressor in colorectal cancer.

Conclusions Our studies uncover a mechanism for constitutive LINC00113/miR-144-5 p/TCF3 feedback loop activation in colorectal cancer. Besides, high expressed of LINC00113 resulted in the down-regulation of STAT1, which further promoted the progression of colorectal cancer.

\section{IDDF2018-ABS-0176 ASSESSMENT OF QUALITY OF LIFE IN ADVANCED GASTRO-INTESTINAL CANCER PATIENTS RECEIVING CHEMOTHERAPY}

Ritu Balal ${ }^{*}$, Harminder Singh. BFUHS, Faridkot, India

\subsection{6/gutjnl-2018-IDDFabstracts.21}

Background The objective of this study is to assess the common gastro-intestinal (GI) symptoms and quality of life (QOL) in advanced GI tract cancer patients with the help of MD Anderson Symptom Inventory (MDASI-GI) validated scale. The objective of this study is to assess the common gastrointestinal (GI) symptoms and quality of life (QOL) in advanced GI tract cancer patients with the help of MD Anderson Symptom Inventory (MDASI-GI) validated scale.

Methods This is a cross-sectional, non-interventional and observational study. A total of 48 patients were interviewed about the GI symptoms and their effect on day to day life with MDASI-GI scale after taking written informed consent from patients.

Results The study population consisted of 23 males and 25 females with an average age of 48.16 years. Among cancer category, $52.08 \%$ were of Upper GI tract cancer. Symptomwise $59.12 \%$ showed mild category of GI symptoms. According to QOL, 65.12\% of the patients mildly affected with reference to day to day activities. Out of a total of 48 patients, 25 had esophageal/upper GI cancer; 10 were of hepatobiliary followed by 8 having colorectal cancer; and 5 with buccal mucosal cancer. 25 patients were treated with Cisplatin/Oxaliplatin with 5-Fluorouracil chemotherapy; 11 patients received Gemcitabine; 8 were given Paclitaxel; and 4 received Methotrexate.

Abstract IDDF2018-ABS-0176 Table 1 Demographic data, type of cancer and symptoms

\begin{tabular}{lll}
\hline Parameters & Number & Percentage \\
\hline Male & 23 & 47.92 \\
Female & 25 & 52.08 \\
Age group (Years) & & \\
$41-50$ & 19 & 39.58 \\
$51-60$ & 07 & 14.58 \\
$61-70$ & 15 & 31.26 \\
$>70$ & 07 & 14.58 \\
Female History & & \\
Present & 09 & 18.75 \\
Absent & 39 & 81.25 \\
Type of Cancer: & & \\
Upper GI & 25 & 52.08 \\
Hepatobiliary & 10 & 20.84 \\
Colorectal & 08 & 16.67 \\
Buccal mucosa & 05 & 10.41 \\
Symptoms: & & \\
Mild (0-3) & & 59.12 \\
Moderate (4-7) & & 33.33 \\
Severe (8-10) & & 07.55 \\
Q0L & & \\
Mild (0-3) & & 11.75 \\
Moderate (4-7) & & \\
Severe (8-10) & & \\
\hline & & \\
\hline
\end{tabular}

